TIPA. Travaux interdisciplinaires sur la parole et le langage

28 | 2012

Linguistique expérimentale

\title{
Quelques apports sur le traitement syntaxique de la forme " alors que »
}

\section{Fanny Lafontaine}

\section{(2) OpenEdition}

Journals

Édition électronique

URL : http://journals.openedition.org/tipa/133

DOI : 10.4000/tipa.133

ISSN : 2264-7082

Éditeur

Laboratoire Parole et Langage

Référence électronique

Fanny Lafontaine, «Quelques apports sur le traitement syntaxique de la forme « alors que » », TIPA. Travaux interdisciplinaires sur la parole et le langage [En ligne], 28 | 2012, mis en ligne le 29 octobre 2012, consulté le 20 avril 2019. URL : http://journals.openedition.org/tipa/133 ; DOI : 10.4000/tipa.133

Ce document a été généré automatiquement le 20 avril 2019

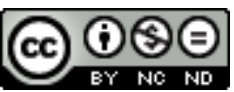

La revue TIPA. Travaux interdisciplinaires sur la parole et le langage est mise à disposition selon les termes de la licence Creative Commons Attribution - Pas d'Utilisation Commerciale - Pas de Modification 4.0 International. 


\title{
Quelques apports sur le traitement syntaxique de la forme " alors que »
}

\author{
Fanny Lafontaine
}

\section{Introduction}

1 Le concept de « subordination circonstancielle », abondamment utilisé dans la grammaire traditionnelle, présente un contenu conceptuel très flou ; Riegel et al. (2008) en donnent la caractérisation suivante :

«[...] les propositions circonstancielles sont introduites par des conjonctions (...) qui à la fois marquent leur caractère subordonné et spécifient leur rapport sémantique avec le reste de la phrase » (475)

2 Cette définition laisse donc entendre qu'une marque morphologique, en l'occurrence, une conjonction, serait à même de déterminer une fonction syntaxique, la subordination, ainsi qu'une relation sémantique. Or, certains grammairiens ont bien repéré qu'on ne pouvait décrire le fonctionnement des conjonctions d'une manière unifiée, mais les divers emplois recensés ont généralement été distingués sur la seule base de leurs effets de signification sans réel souci de dégager leurs propriétés formelles.

Plusieurs études récentes menées sur un certain nombre de conjonctions ${ }^{1}$ mettent en évidence que les séquences ainsi introduites sont susceptibles de relever de plusieurs fonctionnements syntaxiques et ne peuvent être décrites à partir de la simple dichotomie "subordination» vs. "coordination». Ainsi, à partir de données attestées, principalement issues de corpus oraux ${ }^{2}$, nous montrerons que la forme "alors que " possède trois statuts syntaxiques différents associés à des effets de sens particuliers. Pour ce faire, nous aborderons le traitement de cette forme dans la grammaire traditionnelle avant de présenter la méthodologie adoptée. 


\section{La forme alors que dans les analyses traditionnelles}

4 acquis au fil du temps d'autres sémantismes ${ }^{3}$, pour lesquels les grammaires ont des divergences de traitement.

[1] Mes nerfs se sont retrempés à ce souffle marin, plein de sels pénétrants, qui nous frappait la figure alors que nous fendions l'espace, en tilbury découvert lancé au galop. (J. Barbey D’Aurevilly, Premier Memorandum, 1836 : 52).

- et un sens « logique », qui traduit l'idée d'opposition, subdivisé en deux sous-parties :

Un premier cas qui « marque l'opposition sans plus»:

[2] La marraine avait pris un assez fort ascendant sur Ninon, qui avait grand besoin de conseils, alors que la vieille dame en fournissait à foison. (R. Boylesve, La Leçon d'amour dans un parc, 1902: 92).

Un second cas qui « marque la discordance, l'incompatibilité des deux faits que l'on rapproche »:

[3] Mais que peut-il y avoir de commun, au point de vue de la grandeur, entre l'extensif et l'intensif, entre l'étendu et l'inétendu ? Si, dans le premier cas, on appelle plus grande quantité celle qui contient l'autre, pourquoi parler encore de quantité et de grandeur alors qu'il n'y a plus de contenant ni de contenu? (H. Bergson, Essai sur les données immédiates de la conscience, 1889 : 16).

M. Grevisse (2008), quant à lui, ne souligne pas les différents effets de sens soulevés par cette forme en lui attribuant uniquement une valeur temporelle ${ }^{5}$-comme en 1-assortie ou non d'une nuance d'opposition; il cite donc un exemple où les deux domaines sémantiques -le trait « temporel » et « oppositif »- se mêlent :

[4] Les pintades sauvages s'envolaient devant eux, tandis que les perdreaux, écrasés par la chaleur, se contentaient de s'écarter un peu ${ }^{6}(\mathrm{~J}$. \& J. Tharaud, Randonnée de Samba Siouf, p. 103, cité par Grevisse (2008: 602).

Hormis ces divergences de traitement sémantique, la question de la réelle dépendance syntaxique n'est à la limite même plus à débattre puisque tous les grammairiens s'accordent à dire que ces structures ne s'appréhendent que sous le statut de la subordination, lequel est ainsi défini :

«Les propositions subordonnées sont généralement introduites par des termes marquant leur dépendance par rapport à la principale: conjonctions de subordination, pronoms relatifs et mots interrogatifs. » (Riegel et al., $2008: 470$ ).

Autrement dit, ce rapport de dépendance (le lien de subordination) tient à une seule chose : un marquage apparent de la relation syntaxique au moyen d'un terme spécifique. Ce seul critère morphologique, non couplé à d'autres critères formels, n'est pas pour nous 
un indice suffisant pour définir une authentique relation de rection syntaxique ; dans les faits, on peut rencontrer des constructions qui, bien qu'introduites par une « conjonction de subordination", ne dépendent pas du verbe de la construction précédente mais fonctionnent au niveau du discours en permettant d'enchaîner sur une nouvelle énonciation ${ }^{7}$. Ici, à la suite d'autres syntacticiens, nous remettrons en cause ce postulat associant une fonction syntaxique à la présence d'un morphème.

Nous nous fonderons donc sur l'idée qu'un élément morphologique donné peut connaître plusieurs fonctionnements syntaxiques:

«Certains éléments ont pour la même forme lexicale divers emplois possibles: comme régis ou comme associés ; leur sens est alors différent selon ces emplois. »

(Blanche-Benveniste et al., 1990 : 78).

Ainsi, nous montrerons que les différentes valeurs sémantiques de cette conjonction sont liées à une différence de statut grammatical, qui nous amènera à décrire de façon précise la syntaxe externe ${ }^{8}$ de la « alors que construction».

\section{Méthodologie : données et cadre de description}

\subsection{Importance des données}

La place accordée aux données réelles est centrale pour notre étude comme moyen d'approfondir nos connaissances sur ce morphème. Notre démarche consiste donc à prendre l'observation de ces données réelles pour point de départ afin de dégager des critères basés sur des généralisations descriptives qui permettront de rendre compte de la grande variété des fonctionnements syntaxiques et sémantiques de ce morphème. Nous considérons, en effet, que les données sont premières et que c'est la théorie qui doit se construire pour s'adapter aux données. Pour cette étude, nous avons choisi de donner plus d'importance au traitement de productions spontanées. Les données écrites sont là juste pour étayer certains de nos propos. Aussi, pour l'heure, notre objectif n'est pas de présenter une étude quantifiée précise quant aux usages en fonction des types de textes", mais de dégager certains types grammaticaux remarquables par leurs propriétés syntaxiques.

\subsection{Cadre de description}

Le cadre de description qui fondera ce travail délaissera la notion de "subordination ", jugée trop problématique, pour étudier les relations de dépendance syntaxique à partir de la notion de « rection ».

Ainsi, un élément est dit régi (ou construit) par un verbe s'il entretient avec celui-ci un lien de dépendance syntaxique. ${ }^{10}$ Pour rendre compte de cette dépendance, l'approche pronominale ${ }^{11}$ décrit les éléments organisés par le verbe par le recours aux paradigmes de pronoms. Cette réduction présente le double avantage de :

«supprimer les problèmes de sélection lexicale» et fournir «une description grammaticale plus fine que les éléments lexicaux». Ainsi, "pour utiliser systématiquement les pronoms, nous avons dû élargir la liste des éléments qu'on classe généralement sous ce vocable; il vaudrait mieux du reste parler de proformes $^{12}$.» (Blanche-Benveniste et al., $1990: 41$ ).

Un énoncé « chargé » lexicalement comme [5] : 
[5] En 1994, la France adoptait ses premières lois de bioéthique, dans le but de trouver un point d'équilibre entre la protection des droits fondamentaux de la personne et la non-entrave aux progrès de la recherche. (Le Monde, 05/07/2008) paradigme ouvert par la catégorie rectrice. A partir de [5], on peut tester, grâce à ces différents tests d'insertion paradigmatique, le statut syntaxique du constituant introduit par « dans ce but».

\section{d'une classe paradigmatique :}

[5b] c'est dans ce but qu'elle les adoptait et de donner une valeur rhématique à ce qui suit ${ }^{13}$ :

[5e] elle les adoptait et cela dans le but de...

Ces tests permettent de relever au moins deux fonctionnements syntaxiques distincts de l'item qui nous intéresse :

[6] Certains prennent la direction du Val-d'Oise, d'autres sont arrêtés sur le port du Havre alors qu'ils tentent d'embarquer clandestinement sur un bateau en partance pour le Canada. (Le Monde).

[7] les productions qu'on fait à l'écrit c'est vraiment des belles phrases bien construites alors que des productions à l'oral il y a des ben de partout des euh de partout euh (ORAL : Corpaix).

Dans l'exemple [6], la construction en "alors que " peut être analysée comme régie car elle possède, entre autres, une équivalence à la proforme «à ce moment-là ». Elle entre alors en concurrence avec des morphèmes comme « lorsque » ou « quand ». En revanche, dans [7], malgré la présence d'une " conjonction de subordination », la séquence n'a pas de relation de dépendance avec ce qui précède car elle ne répond pas aux différents tests de l'approche pronominale. Ici, elle ne possède aucune équivalence paradigmatique avec 
d'autres conjonctions, mais peut être mise en relation avec différents connecteurs « adversatifs », tels que « par contre » ou « en revanche».

Les trois emplois que nous proposons de distinguer vont être décrits ci-dessous.

\section{Les trois types de " alors que »}

\subsection{Type I : marqueur d'une rection temporelle}

On peut illustrer ce type à partir de cet exemple cité par M. Grevisse (2008: 1482) :

[8] Je l'ai vue deux ou trois fois alors que j'étais à Toulouse.

Dans cet usage, nous faisons de la construction verbale introduite par la forme alors que un élément régi, à statut d'ajout qui serait analysable comme une "subordonnée " classique pourvue d'une valeur temporelle dominante, où notre forme entre dans un paradigme de conjonctions, lorsque et quand :

[8a] Je l'ai vue deux ou trois fois lorsque/quand j'étais à Toulouse

La relation de dépendance entre le verbe recteur et la « alors que construction » est ainsi confirmée par l'équivalence à une proforme :

[8b] Je l'ai vue deux ou trois fois à ce moment-là

Le test de la reprise par « et ce » est aussi convaincant :

[8c] Je l'ai vue deux ou trois fois et ce alors que j'étais à Toulouse

On observe en outre que, contrairement à d'autres emplois qui seront décrits plus bas, ce type de construction est mobile et peut donc apparaître postposée à la première construction verbale comme en [8], ou antéposée :

[9] Alors que leurs employeurs n'ont toujours pas versé le premier centime de cotisations, payables en avril, les gardes d'enfants trinquent déjà. (Le Nouvel Observateur)

Si une partie des tests corroborent bien ce lien de dépendance syntaxique entre le verbe recteur et la construction introduite par "alors que », en revanche les manipulations avec d'autres tests rendent certains énoncés peu naturels. C'est le cas avec l'interrogation sur les circonstances [8d], le clivage [8e], le contraste de modalités [8f] et l'insertion d'un adverbe paradigmatisant $[8 \mathrm{~g}]$ :

[8d] ? Quand l'as-tu vue deux trois fois ? Alors que j'étais à Toulouse

[8e] ? C'est alors que j'étais à Toulouse que je l'ai vue deux ou trois fois

[8f] ? Je l'ai vue deux ou trois fois non pas alors que j'étais à Toulouse mais alors que j'étais à Paris 
[8g] ? Je l'ai vue deux trois fois probablement alors que j'étais à Toulouse

subordination " à valeur temporelle dominante malgré les blocages aux tests qui
semblent inhérents à cette forme. On peut relever le fait que ce sémantisme temporel est
extrêmement minoritaire dans les données orales, d'où nos doutes sur la recevabilité de
certains énoncés.

\subsection{Type II : emploi de « connexion discursive »}

\subsubsection{Les propriétés syntaxiques}

A partir de l'exemple de corpus suivant, nous reproduisons les mêmes tests que ceux utilisés précédemment afin de vérifier si la construction introduite par « alors que » est liée syntaxiquement au verbe de la construction précédente :

[10] sur l'Acropora c'est un petit polype alors que sur le Catalaphyllia - c'est des c'est des c'est des gros polypes (oral, CRFP).

Il n'existe pas d'équivalence à une proforme: aucun pronom ne peut représenter cette construction verbale.

Le clivage ne fonctionne pas :

[10a] ${ }^{* 16}$ c'est alors que sur le Catalaphyllia - c'est des c'est des c'est des gros polypes que sur l'Acropora c'est un petit polype 
On ne peut y insérer un adverbe paradigmatisant :

[10b] * sur l'Acropora c'est un petit polype surtout alors que sur le Catalaphyllia c'est des c'est des c'est des gros polypes

L'absence d'appartenance à un paradigme induit qu'on ne peut pas non plus l'organiser en couples contrastifs :

[10c] * sur l'Acropora c'est un petit polype alors que sur le Catalaphyllia - c'est des c'est des c'est des gros polypes et non alors que...

Elle ne supporte pas le détachement, comme le montre l'impossibilité d'avoir une reprise en « et ce» :

[10d] * sur l'Acropora c'est un petit polype et ce alors que sur le Catalaphyllia - c'est des c'est des c'est des gros polypes

Comme notre exemple ne répond pas aux propriétés des éléments régis, la construction introduite par alors que ne peut être ramenée à des faits de dépendance grammaticalemais plutôt à des faits qui mettent en jeu des structures discursives et textuelles. Dans cet usage, chaque construction verbale possède sa propre autonomie syntaxique si bien que le fonctionnement de alors que doit être saisi comme un phénomène de combinaisons entre constructions et non comme un phénomène où la structure serait enchâssée à l'autre construction.

On pourrait considérer que la relation entre les deux constructions relève ici d'un fonctionnement paratactique, dans lequel le morphème- permettant d'assurer le rattachement entre les deux unités autonomes successives- est plus proche du fonctionnement d'un connecteur à valeur discursive que d'une conjonction. On le vérifie notamment en constatant une équivalence avec certains connecteurs discursifs qui possèdent une valeur adversative dominante tels que par contre, en revanche, mais ${ }^{17}$.

[10e] sur l'Acropora c'est un petit polype par contre/ mais/ en revanche sur le Catalaphyllia - c'est des c'est des c'est des gros polypes

K. Sandfeld (1965 : 305) relève cette autonomie syntaxique : «fréquemment, la proposition adversative se détache et devient indépendante, de sorte que 'tandis que', 'alors que'..., se rapprochent du sens de 'par contre'. ».

De plus, contrairement aux emplois régis d'ajout temporel qui jouissent d'une certaine mobilité (cf. ex. 9), ici, la séquence introduite par « alors que » se situe invariablement à droite de l'autre construction verbale; le second membre prend nécessairement appui sur le premier. Dans les données, on ne rencontre pas d'emplois comme :

[10f] ? alors que sur le Catalaphyllia - c'est des c'est des c'est des gros polypes sur l'Acropora c'est un petit polype

Cette contrainte sur la distribution des unités ${ }^{18}$ s'explique si on considère que ce morphème introduit une nouvelle unité de discours comme le feraient les différents connecteurs adversatifs dont il est proche : 
$[10 \mathrm{~g}]^{*}$ Par contre sur le Catalaphyllia - c'est des c'est des c'est des gros polypes sur l'Acropora c'est un petit polype

[10h] * En revanche sur le Catalaphyllia - c'est des c'est des c'est des gros polypes sur l'Acropora c'est un petit polype

Donc, en d'autres termes, plus pragmatiques, chaque construction verbale introduit un nouvel acte illocutoire, en l'occurrence deux assertions, dans notre exemple :

[10i] [sur le sur l'Acropora c'est un petit polype] alors que [sur le Catalaphyllia - c'est des c'est des c'est des gros polypes]

K. Sandfeld, (1965: 306) énonce un exemple ${ }^{19}$ dans lequel on constate encore plus nettement que la seconde construction peut avoir sa propre force illocutoire, comme le montre le passage de la modalité assertive à jussive :

[11] A ce jeu de voleurs, les chrétiens ne sont pas de force, ils finissent toujours par se noyer ; tandis que prenez un juif... jettez-le dans l'eau trouble de quelque affaire véreuse, et il se sauvera. ( $\mathrm{Z}$. Argent 93 )

\subsubsection{Quelques phénomènes discursifs particuliers}

51 Ce type de structure se singularise en grande partie par la présence de propriétés formelles remarquables, que l'on rencontre très fréquemment dans les corpus. ${ }^{20}$

\section{a/ Les effets de parallélisme syntaxique}

Les constructions en «alors que » développent certains effets de parallélisme syntaxique ${ }^{21}$ plus aptes à faire ressortir les phénomènes d'oppositions lexicales présents sur deux paradigmes. En effet, Blanche-Benveniste et al., (1990 : 181) expliquent :

«lorsque, dans deux énoncés successifs, on retrouve le même schéma syntaxique, c'est souvent avec un effet de contraste; les lexèmes placés dans les mêmes positions syntaxiques contrastent entre eux. ».

[12] moi je jouais dans un orchestre elle elle chantait euh elle dans les cours (Vie parisienne).

Comme le parallélisme, le chiasme est une configuration syntaxique fondée sur la symétrie de structures. Dans la première construction verbale, le thème se trouve à la droite du verbe recteur, dans celle introduite par «alors que », il se trouve à sa gauche, comme dans :

[13] L1 des des enzymes contenues dans le le venin des crochets justement il y a quand même une action venimeuse mais bon elle est pas elle est pas significative chez les espèces américaines hein L2 oui L1 alors que chez euh chez des asiatiques comme celle-ci c'est elle est p- elle est prépondérante (NCY-R00PUB001) 

obligatoirement la présence d'un connecteur. des c'est des gros polypes et que nous appelons « pré-noyaux $»^{22}$.

Plus précisément, Charolles (Ibid : 7) écrit que ces cadratifs pas instanciés dans le texte. » symétriques. le sens : l'Acropora c'est un petit polype

\section{b/ Le phénomène de projection syntaxique} décrit ces projections comme :

Elle cite des exemples du genre (151) : jamais (CF 4,8).

Dans ces emplois fortement symétriques, « alors que » peut également commuter avec 0 , c'est-à-dire qu'il est possible de le supprimer sans faire disparaître l'effet de contraste ; l'opposition peut simplement être exprimée par le lexique et ne nécessite pas

[10j] sur le sur l'Acropora c'est un petit polype sur le Catalaphyllia - c'est des c'est

Les travaux de Charolles (1997) sur « l'encadrement du discours » éclairent notre travail sur la présence de ces éléments qui apparaissent en tête de chaque construction verbale

«mettent essentiellement en jeu une relation de contraste. Préciser qu'une proposition (p) est vérifiée sous une circonstance $C$ ou, plus généralement, relativement à un certain critère $C$, qu'elle n'est vraie que sous $C$, ce qui revient à évoquer toutes les autres circonstances dans lesquelles elle ne serait pas vraie. L'instanciation d'un univers de discours projette donc, par inférence locale, ce que nous appellerons un ensemble d'univers parents (correspondant à l'ensemble des circonstances différentes de C), univers qui demeurent virtuels tant qu'ils ne sont

La forme "alors que " est sujette à développer ces phénomènes de "projections sémantiques» qui se réaliseront bien souvent dans des structures syntaxiques

Aussi, il est possible de renverser les deux constructions verbales sans que cela ne change

[10k] sur le Catalaphyllia - c'est des c'est des c'est des gros polypes alors que sur

Cet usage de « alors que » est pour la plupart du temps compatible avec les structures à projection, qui correspondent à une « organisation lexicale bis de la relation syntaxique donnée préalablement » (Blanche-Benveniste et al., 1990 : 152). C. Blanche-Benveniste (ibid. : 157)

«des structures apparentées aux listes; elles manifestent cette propriété paradigmatique de réitération, mais elles engagent des relations plus complexes, qui donnent une plus grande indépendance aux éléments listés » .

[14] Tu vois cette mer elle se lave deux fois deux fois par jour la Méditerranée

Ce phénomène de projection s'instaure par la présence d'«au moins deux éléments appartenant à deux paradigmes différents de la construction verbale. » (ibid. : 152).

Ainsi, on pourrait, dans ce type d'usage, simplifier la syntaxe interne ${ }^{23}$ de la construction introduite par « alors que » à deux éléments, l'un réalisant une place syntaxique et l'autre 
une modalité de relation qui s'interpréterait relativement à ce qui précède, c'est-à-dire par référence aux positions syntaxiques ouvertes dans la construction verbale de gauche ; à partir de notre même exemple, en projetant la seconde construction sur la première, on aurait cette équivalence avec l'élément locatif là et la modalité de relation non :

[10l] sur l'Acropora c'est un petit polype alors que là non

Voici un exemple concernant la différence entre « petites et grandes surfaces $»^{24}$ :

[15] donc je peux pas euh baisser les prix sans gagner ma vie c'est pas possible - c'est pas possible je suis obligée d'avoir une marge bénéficiaire - tandis que les grandes surfaces non - (CRFP)

A l'intérieur de la construction en "alors que ", on a donc un regroupement de type «pré-noyau/ noyau ».

Le sémantisme négatif induit par la forme "alors que» oblige nécessairement la réitération d'une modalité négative si le premier membre est assertif :

$[10 \mathrm{~m}]$ * sur le sur l'Acropora c'est un petit polype alors que sur le Catalaphyllia aussi

Contrairement à des exemples où l'on aurait :

[16] eux les prononcent nous aussi (ibid. : 156).

67 On pourrait même envisager un exemple comme le suivant, dans lequel ce schéma syntaxique rend possible l'élision du noyau du second membre, que l'on pourrait reconstituer par le jeu des contrastes lexicaux :

[17] Paul est un as de la guitare alors que Alain... (exemple inventé). du type «j'ai ri »-par opposition à «j'ai pleuré »- or, l'effet comique naît du décalage entre cette « attente logique » et ce qui est véritablement instancié.

\section{c/ Les faits de « séquencialité "}

71 Enfin, on peut relever quelques faits de «séquencialité", qui correspondent à l'enchaînement de plusieurs constructions introduites par le morphème "alors que ", sans qu'il y ait interchangeabilité entre elles et où chaque construction prend appui sur celle qui la précède : 
[19] là on se situe euh - donc devant un bac qu'on appelle récifal - parce qu'en fait il est en comparaison avec d'autres bacs d'eau de mer celui-là il a une prépondérance il a le comment - il y a une prépondérance de coraux - alors que dans les autres bacs c'est essentiellement euh des poissons - ou euh quelques échinodermes ou des des crustacés hein disons style oursin(s) euh - concombre(s) de mer - alors que dans celui-là euh - il est peuplé en majeure partie de de coraux les poissons sont pas absents puisque il y en a puisqu'ils servent aussi à - à réguler l'ensemble euh ils interviennent dans l'équilibre (NCY-R00PUB001)

Ces séquences, qui peuvent développer jusqu'à trois constructions en "alors que ", présentent des particularités :

-on ne peut inverser les deux constructions en « alors que » :

[19a] * celui-là il a une prépondérance il a le comment - il y a une prépondérance de coraux - alors que dans celui-là euh - il est peuplé en majeure partie de de coraux alors que dans les autres bacs c'est essentiellement euh des poissons

-on ne peut non plus supprimer la première :

[19b] * celui-là il a une prépondérance il a le comment - il y a une prépondérance de coraux - alors que dans celui-là euh - il est peuplé en majeure partie de de coraux

Jeanne Marie Debaisieux (2007) observe des cas similaires avec le morphème « puisque » :

[20] janvier c'est là où tout est concentré euh les inséminations sont concentrées puisque les éleveurs cherchent à à à optimiser le le la production laitière de chaque vache puisque le lait euh est bien payé à la période d'août août septembre octobre.

Selon elle, "ces fonctionnements non régis présentent des propriétés 'quasi distributionnelles'. Les constructions sont soumises à des contraintes d'apparition qui les distinguent d'autres cas. »

Ces structures ne sont donc pas dépendantes syntaxiquement du verbe de la première construction, comme nous l'avons montré par les tests de la rection. De plus, elles manifestent des relations symétriques et paradigmatiques basées sur des phénomènes d'oppositions lexicales qui en font un type particulier On peut dire qu'ici « alors que » a un fonctionnement de "connecteur discursif» particulier que l'on peut caractériser comme « adversatif $»^{25}$.

\subsection{Type III : introducteur d'un élément associé à une construction}

\subsubsection{Les distinctions par rapport à l'usage précédent de « connecteur discursif »}

Bien que K. Sandfeld mentionne que, dans cet usage, alors que "ne differe pas toujours nettement de l'emploi qu'il a dans des propositions adversatives" (1965: 328), nous voulons rendre compte, par certaines propriétés, qu'il s'agit d'un type de non régi distinct des exemples étudiés précédemment et non assimilable à un fonctionnement de connecteur.

Ainsi, à partir de l'exemple 21,

[21] je comprends pas qu'on dise que Gutenberg a inventé l'imprimerie alors qu'il travaillait dans une imprimerie (CRFP) 
81

alors que n'entre pas dans un paradigme d'éléments adversatifs tels que par contre :

[21a] * je ne comprends pas qu'on dise que Gutenberg a inventé l'imprimerie par contre/mais il travaillait dans une imprimerie

En outre, K. Sandfeld fait un rapprochement entre certains emplois introduits par puisque et d'autres par alors que qu'il classe donc parmi les « propositions causales »:

«On se sert aussi d'une proposition temporelle introduite par quand, lorsque ou alors que. Si l'emploi de puisque veut dire, primitivement, qu'on demande comment un fait peut exister après un autre qui devrait l'exclure, l'emploi de quand et alors que marque qu'on demande comment un fait peut coexister avec un autre.» (ibid. : 328).

[21b] je ne comprends pas qu'on dise que Gutenberg a inventé l'imprimerie puisqu'il travaillait dans une imprimerie

Puisque n'est pas la seule forme "causale » qui puisse être substituée à alors que; C. Guimier (2000) considère que dans un tel cas " 'alors que' est glosable par 'étant donné que'" (98).

[21c] je comprends pas qu'on dise que Gutenberg a inventé l'imprimerie étant donné qu'il travaillait dans une imprimerie

De même, vu que semble lui être également substituable ${ }^{26}$ :

[21d] je comprends pas qu'on dise que Gutenberg a inventé l'imprimerie vu qu'il travaillait dans une imprimerie

Enfin, alors que ne peut être rapproché, dans cet usage, d'un fonctionnement de connecteur car il ne met pas en jeu les mêmes relations paradigmatiques. En effet, précédemment, les phénomènes d'oppositions lexicales se jouaient sur deux paradigmes et l'on observait un changement de thème qui se manifestait souvent par la présence d'un «cadratif ». Ici, dans notre exemple, le même thème "Gutenberg » est repris dans la construction introduite par «alors que » par le clitique « il». Ainsi, il est plus délicat de savoir si l'on a affaire à un ou deux actes illocutoires.

\subsubsection{Sur le caractère régi ou non}

\section{a/ Les tests de la rection}

L'opposition fondamentale entre élément régi/non régi pose ici des difficultés avec l'application des tests syntaxiques. En effet, à partir de cet exemple (21), les tests d'insertion paradigmatique, (le clivage (e), l'insertion d'un adverbe (f) et le contraste de modalités (g)), montrent qu'il n'y a pas de relation de rection entre le verbe recteur et la construction introduite par « alors que » :

[21e] * c'est alors qu'il travaillait dans une imprimerie que je comprends pas qu'on dise que Gutenberg a inventé l'imprimerie

TIPA. Travaux interdisciplinaires sur la parole et le langage, 28 | 2012 
[21f] * je comprends pas qu'on dise que Gutenberg a inventé l'imprimerie surtout alors qu'il travaillait dans une imprimerie

[21g] * je comprends pas qu'on dise que Gutenberg a inventé l'imprimerie non pas alors qu'il travaillait dans une imprimerie mais alors qu'il...

Mais si une partie de ces tests d'insertion paradigmatique ne fonctionne pas, il semblerait toutefois qu'on ait une équivalence entre la «alors que construction» et la proforme « dans ces conditions-là $»^{27}$ :

[21h] je comprends pas qu'on dise que Gutenberg a inventé l'imprimerie dans ces conditions-là.

Aussi, la reprise par « et cela » semble acceptable :

[21i] ? je comprends pas qu'on dise que Gutenberg a inventé l'imprimerie et cela alors qu'il travaillait dans une imprimerie

Ainsi, si on considère cette résistance à l'application de certains tests syntaxiques et le fait que cet usage ne relève pas d'un fonctionnement de connecteur, on pourrait présenter cette relation entre le verbe et la "alors que construction" comme une relation d'association. Nous entendons par "associés", "les éléments qui ont parfois l'apparence de compléments régis par le verbe, mais qui n'en sont pas. Ils n'ont aucune des propriétés des éléments régis» (Blanche-Benveniste et al., 1990: 77). Ces éléments se retrouvent ainsi enchaînés à d'autres sans lien de rection pour former un énoncé complet s'apparentant à une « phrase ».

\section{b/ Une relation particulière de regroupement}

Dans tous les exemples relevant de ce fonctionnement, on rencontre deux types de phénomènes : d'une part, des cas d'enchâssement où la « alors que construction » est soit enchâssée à un verbe ou soit saisie par le clitique « ce » et d'autre part, des faits de portée large d'une interrogation ou d'une négation. On a ainsi une forme de regroupement particulier qui s'instaure entre la « alors que construction » et le contexte précédent.

91 La portée sémantique de la négation et de l'interrogation.

92 Comme indice de ce regroupement, on peut relever le fait que la construction introduite par « alors que » semble être atteinte par la portée de l'interrogation ou de la négation.

Dans des exemples d'interrogation directe, comme 22 :

[22] Comment les riches Parisiens peuvent-ils jouir gaiement de leur luxe, alors que, pour quitter la ville ils doivent traverser quinze kilomètres d'une banlieue sordide?

(Duh. Querelles 43, cité par Sandfeld, 1965 : 328).

il n'est pas aisé de savoir si la "alors que construction" est sous la portée de l'interrogation ou non et donc si l'ensemble forme une ou deux unités. L'argument intonatif semble corroborer cette intégration syntaxique puisque les locuteurs ont tendance à énoncer ces deux constructions verbales d'un seul tenant. 


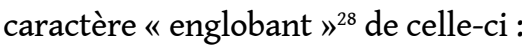
appris pendant deux ans - (PSO-R0OPRO001)

- Les problèmes de l'enchâssabilité. à cinq cents mètres l'un de l'autre même pas (CRFP) plainte est déposée au commissariat ou autre quoi (Corpaix)

Le cas de puisque.

Ainsi, à partir de [26] : "comprend" un langage "abstrait". (DIVS.Philo) «pourquoi »: dauphin "comprend" un langage "abstrait"

Elle ne peut être mise sous clivage : qu'il s'agit d'un comportement intelligent dauphin "comprend" un langage "abstrait"

De même, dans les structures contenant une négation, on peut s'interroger sur le

[23] L1 parce que maintenant les gens> les gens tu vois il faut il faut - enfin ils vont pas non plus passer du temps à former alors que il y a des gens qui vont sortir d'une école qui ont fait pendant deux ans - euh qui ont fait ça pendant deux ans qui ont

On sait que les phénomènes d'enchâssement sont en principe à considérer comme une forme de dépendance syntaxique entre deux constructions. Dans cet usage, ceux-ci sont susceptibles de se rencontrer dans deux types de structures, l'interrogation indirecte, comme [21] et les structures du type « c'est + adjectif évaluatif » :

[24] c'était un peu idiot de refaire d'essayer de refaire la même chose alors qu'on est

[25] il y va de soi que ce serait bête de passer à côté d'une affaire alors qu'une :

Si on considère que dans ces emplois le fonctionnement de "alors que " se rapproche de celui de "puisque», alors les études menées sur puisque par le groupe lambda (1975) et plus tard par J. M. Debaisieux (2006) ont montré que les constructions en puisque «ne participent qu'à un seul fonctionnement non assimilable à une relation de rection », et ce malgré la présence d'un verbe susceptible d'enchâsser, comme « déduire ».

[26] On en déduit qu'il s'agit d'un comportement intelligent puisque le dauphin

La construction introduite par «puisque» n'a pas d'équivalence avec la proforme

[26a]* On en déduit qu'il s'agit d'un comportement intelligent pourquoi ? Puisque le

[26b] * C'est puisque le dauphin "comprend" un langage "abstrait" qu'on en déduit

Elle ne peut non plus être modifiée par un adverbe paradigmatisant :

[26c] * on en déduit qu'il s'agit d'un comportement intelligent seulement puisque le 

syntaxique :

[21m] ce que je ne comprends pas c'est qu'on dise que Gutenberg a inventé l'imprimerie alors qu'il travaillait dans une imprimerie

Le verbe « déduire ", susceptible d'enchâsser, ne prend pas dans sa portée la construction introduite par puisque :

[26d] on en déduit cela puisque le dauphin "comprend" un langage "abstrait"

D'où la possibilité d'antéposer la séquence en puisque :

[26e] puisque le dauphin "comprend" un langage "abstrait" on en déduit cela

On voit donc que la construction introduite par « puisque » est « sentie comme l'explication ou la justification» (groupe lambda, $1975: 252$ ) de la première construction verbale et qu'elle ne peut donc être enchâssée. Ainsi, « les énoncés de type $p$ puisque q comportent deux actes successifs: l'un consiste à énoncer $p$, l'autre à présenter, en affirmant $q$, une sorte de justification du premier.» (ibid. : 275).

Donc, d'après les travaux, les éléments non régis ne semblent pas avoir la possibilité d'être enchâssés.

Le cas de alors que :

Or, dans l'exemple [21], la «alors que construction » ainsi que la construction verbale introduite par le verbe "dire " semblent être enchâssées au verbe "comprendre ", comme le montre la possibilité d'englober toute la séquence par « cela » :

[21j] Je ne comprends pas cela.

Pour corroborer cela, on ne pourrait pas avoir de pronominalisation en «ça » ou « le » qui n'englobe que la séquence introduite par le verbe « dire » :

[21k] ? je ne comprends pas ça alors qu'il travaillait dans une imprimerie

[211] ? je ne le comprends pas alors qu'il travaillait dans une imprimerie

Ces séquences seraient recevables mais elles ne contiendraient pas la même valeur axiologique que la séquence originale.

On en déduit que ce qui doit être saisi par le pronom (le ou ça), c'est l'ensemble de la séquence, y compris la « alors que construction ».

En fait, l'ensemble du regroupement semble constituer, dans cet exemple, une réalisation particulière de la rection.

Aussi, le test de la pseudo clivée ${ }^{29}$ nous fournit l'indice d'une forme d'intégration

Il nous semble, ici, que le clitique «ce » prend bien dans sa portée la construction en « alors que ».

6 On le voit par l'impossibilité d'avoir la séquence en «alors que » en position de prénoyau : 
[21n]? alors qu'il travaillait dans une imprimerie, ce que je ne comprends pas c'est qu'on dise que Gutenberg a inventé l'imprimerie

117 Donc par son intégration dans une pseudo-clivée, ce type de "alors que " ne semble développer qu'un seul acte illocutoire.

\subsubsection{Des conditions pragmatiques particulières : la valeur polémique}

118 Ce qui caractérise ces emplois, c'est que la «alors que construction » apparaît toujours dans un contexte "polémique" où un lexique axiologique fournissant un caractère évaluatif est utilisé ainsi qu'une marque morphosyntaxique, la négation ${ }^{30}$. Aussi, le type d'interrogation dans lequel intervient cette structure n'est pas une interrogation " ordinaire » demandant un complément d'information, mais une interrogation oratoire, comme l'appelait la rhétorique, qui « oriente l'interlocuteur vers une assertion déterminée. Affirmative ou négative, elle implique le contraire de ce qu'exprime leur fonction grammaticale: quand elle est affirmative, elle nie, quand elle est négative, elle affirme.» (Riegel et al., 2008: 400-401).

En effet, sous la forme d'une «fausse question » ou d'une évaluation négative, le locuteur paraît aller à l'encontre d'une opinion ou d'un fait établi, en entrant dans une sorte de polémique. Par exemple, dire «je comprends pas qu'on dise que Gutenberg a inventé l'imprimerie alors qu'il travaillait dans une imprimerie », c'est en quelque sorte mettre en doute l'idée généralement admise que Gutenberg puisse avoir, à strictement parler, inventé l'imprimerie. Dire «c'était un peu idiot d'essayer de refaire la même chose alors qu'on est à cinq cents mètres l'un de l'autre ", c'est mettre en cause l'intérêt qu'il y aurait à « refaire la même chose ».

120 Aussi, à partir de [21], nous avons pu remarquer que si on abandonnait la portion de l'énoncé portant la polémique il se produisait un changement de statut de la «alors que construction »:

[21o] Gutenberg a inventé l'imprimerie alors qu'il travaillait dans une imprimerie

A partir des tests, on retrouverait un « alors que » régi temporel :

[21p] c'est alors qu'il travaillait dans une imprimerie qu'il a inventé l'imprimerie

[21q] Gutenberg a inventé l'imprimerie à ce moment-là

Comme pour les emplois régis, l'antéposition sous forme de pré-noyau ne poserait aucun problème :

[21r] alors qu'il travaillait dans une imprimerie, Gutenberg a inventé l'imprimerie

Ainsi, on remarque que pour déclencher cette valeur polémique, des conditions grammaticales doivent être remplies.

On voit donc avec ces diverses propriétés qu'il n'est pas aisé d'accorder un statut syntaxique net à cet usage à cheval entre deux statuts syntaxiques opposés; le fonctionnement de alors que, ici, ne peut être rapproché de celui de connecteur de 
discours car il ne présente pas les mêmes propriétés Aussi, certains indices nous orientent-ils vers un fonctionnement régi, sans que cela soit tout à fait déterminant. C'est pour ces raisons que nous préférons être prudente et dire que la structure introduite par «alors que » est associée au verbe recteur. Les effets de regroupement que nous avons mis en valeur par la portée sémantique et l'enchâssement renforcent cet effet d'association. Enfin, sur le plan pragmatique, cet usage présente une valeur polémique évidente liée au lexique ou à la structure syntaxique de l'énoncé.

\section{Conclusion générale}

Cette étude nous incite, une fois de plus, à avoir plus de méfiance à l'égard des grandes étiquettes usuelles qui, par le seul argument du statut catégoriel de l'élément introducteur, rangent sous la même dénomination des constructions qui n'ont cependant pas les mêmes propriétés syntaxiques et occultent ainsi certains fonctionnement intéressants.

Pour l'heure, nous ne pouvons prétendre effectuer un traitement quantitatif des données analysées car nous n'en sommes encore qu'à leur recueil. Aussi, notre but n'était pas de rendre compte de l'ensemble des fonctionnements syntactico-sémantiques de cette forme mais simplement de signaler l'existence de cette pluralité de fonctionnements.

Nous avons tenté, ici, de mettre à jour trois fonctionnements syntaxiques distincts qui recouvraient des sémantismes différents ; la dichotomie entre les deux premiers emplois nous a conduite à préciser les limites de la syntaxe de rection. Concernant le type III, nous avons pu voir que «alors que» résistait à certains tests et qu'il présentait des particularités syntactico-pragmatiques intéressantes qu'il faudrait encore affiner. Ainsi, si le recours à la distinction entre régi et non régi s'avère fructueux - on pense notamment à parce que -, en revanche, pour d'autres cas (ici la forme « alors que»), il ne résout pas tous les problèmes.

\section{BIBLIOGRAPHIE}

Bat-Zeev Shyldkrot, H. (1987) Quand, alors que et tandis que : un cas classique d'évolution sémantique, Romance Notes, 28, p. 45-50.

Béguelin, M.-J. (2002) Clause, période ou autre ? La phrase graphique et la question des niveaux d'analyse, Verbum, XXIV, p. 85-107.

Benzitoun, C. (2006) Examen de la notion de « subordination ». Le cas des quand « insubordonnés », Faits de langues, 28, p. 35-46.

Benzitoun, C. (2010) Quelles(s) unité(s) syntaxique(s) maximale(s) en français parlé ? Discussions autour de quelques problèmes rencontrés, Travaux de linguistique, 60, p. 109-126.

Berrendonner, A. (2002) Les deux syntaxes, Verbum, XXIV, p. 23-35. 
Bilger, M. \& P. Cappeau (2010) Une conjonction qui subordonne rarement : le cas de alors que, Actes du colloque international morphologie, syntaxe, et sémantique des subordonnants, ClermontFerrand, mars 2010.

Blanche-Benveniste, C. (1982) Examen de la notion de subordination, RSFP, 4, p. 71-115.

Blanche-Benveniste, C., Deulofeu, J., Stéfanini, J., van den Eynde, K. (1984) Pronom et syntaxe. L'approche pronominale et son application au français, Paris : SELAF, $245 \mathrm{p}$.

Blanche-Benveniste, C., Bilger M., Rouget C., Van den Eynde K., Mertens P. (1990) Le Français parlé: études grammaticales, Paris : éd. du CNRS, 292 p.

Blanche-Benveniste, C. (2002) Phrase et construction verbale, Verbum, XXIV, p. 8-22.

Blumenthal, P. (1980) La syntaxe du message. Application au français moderne, Tübingen : Max Niemeyer Verlag, 206 p.

Bolinger, D. (1977) Another glance at main clause phenomena, Language, 53, 3, p. 511-519.

Charolles, M. (1997) L'encadrement du discours. Univers, champs, domaines et espaces, Cahiers de recherche linguistique, 6, p. 1-73.

Charolles, M. (2003) De la topicalité des adverbiaux détachés en tête de phrase, Travaux de linguistique, 47, p. 11-51.

Culicover, P. \& R. Jackendoff (1997) Semantic Subordination despite Syntactic Coordination, Linguistic Inquiry, 28, 2, p. 195-217.

Danjou-Flaux, N. (1993) Au contraire, connecteur adversatif, Cahiers de linguistique française, 5, p. 275-303.

Debaisieux, J.-M. (2006) Quel statut syntaxique pour les propositions « subordonnées circonstancielles » ? La distinction entre dépendance grammaticale et dépendance macrosyntaxique comme moyen de résoudre les paradoxes de la subordination, Faits de langues, 28 , p. 119-132.

Deulofeu, H.- J. (2001) La notion de construction corrélative en français : typologie et limites, Recherches sur le français parlé, 16, p. 103-124.

Ducrot, O. (1980) Les échelles argumentatives, Paris : Minuit, 96 p.

Ducrot, O. (1983) Puisque : essai de description polyphonique, Revue Romane, 24, p. 166-185.

Ducrot, O. (1993) Opérateurs argumentatifs et visée argumentative, Cahiers de linguistique française, 5, p. 7-36.

Franckel, J.-J. (1986) Alors - alors que, BULAG, 13, p. 17-49.

Gettrup, H. \& H. Nolke (1984) Stratégies concessives : une étude de six adverbes français, Revue romane, 19, 1, p. 3-47.

Green, G. (1976) Main clause phenomena in subordinate clauses, Language, 52, 2, p. 382-397.

Grevisse, M. \& A. Goosse (2008), Le bon usage, 14ème édition, Gembloux : Duculot, 1482 p.

Groupe Lambda-l (1975) Car, parce que, puisque, Revue Romane, X, 2, p. 248-280.

Guimier, C. (2000) Non congruence et congruence : alors que vs. tandis que, Syntaxe et sémantique, 1, p. 79-111.

Nolke, H. (2002) Pour un traitement modulaire de la syntaxe transphrastique, Verbum, XXIV, p. $179-192$. 
Piot, M. (1988) Coordination-subordination : Une définition générale, Langue française, 77, p. 5-18.

Piot, M. (1993) les connecteurs du français de France, Linguisticae Investigationes, 17, 1, p. 142-160.

Riegel, M. et al. (2008) Grammaire méthodique du français, Paris : PUF (5 $5^{\mathrm{ème}}$ édition), 646 p.

Sancier-Château, A. \& D. Denis (1997) Grammaire du français, Paris : Le livre de poche, 541 p.

Sandfeld, K. (1977/1965) Syntaxe du français contemporain. Les propositions subordonnées, Genève :

Droz, $490 \mathrm{p}$.

Van Den Eynde, K. \& P. Mertens (2003) La valence : l'approche pronominale et son application au lexique verbal, Journal of French Language Studies, 13, p. 63-104.

Verstraete, J.-C. (2007) Rethinking the coordinate-subordinate dichotomy. Interpersonnal grammar and the analysis of adverbial classes in English, Berlin: Gruyter Mouton, $321 \mathrm{p}$.

Wilmet, M. (2007) Grammaire critique du français, Bruxelles : Duculot (4ème édition), 758 p.

\section{ANNEXES}

Le recueil des données, qui a permis de mettre à jour ces différents fonctionnements syntaxiques en lien avec l'interface sémantique, s'est effectué sur plusieurs bases de données informatisées rassemblant divers genres ou situations communicationnelles.

Pour la partie orale, nous avons interrogé quatre bases de données :

- Le Corpus de Référence du Français Parlé (CRFP), réalisé par l'équipe DELIC, comporte 440000 mots, soit environ 36 heures de parole. Il comprend des séquences de récits de vie, de souvenirs d'enfance, de prise de parole professionnelle en public ou encore d'explications d'un savoir-faire.

Ce corpus a pris en compte trois critères :

-géographique : Paris et 37 villes de Province

-niveau d'étude des locuteurs : encodage de 0 à 3.

-trois situations de parole : parole privée, professionnelle et publique.

Il nous fournit 115 séquences introduites par le morphème « alors que ».

- Le CRFP II a été réalisé par A. Valli. Il contient environ 20 heures de retranscriptions de l'émission radiophonique France Culture. Il traite de thèmes de société, d'art, de politique... et contient de la parole de « spécialistes » des domaines abordés. Nous avons rencontré 44 séquences avec la forme « alors que ».

- Le Corpus Evolutif de Référence du Français (CERF) est composé de 10 tranches de 1 million de mots chacune dont une d'oral, Corpaix, transcrite par le Gars entre 1990 et 2000. Ce corpus regroupe différentes situations communicationnelles comme des conversations spontanées, des récits de vie ou d'accidents, des situations de parodies, ou encore des témoignages de personnes parlant au nom d'une association. Ce corpus contient 385 séquences en « alors que ».

- Le Corpus de Langues parlées en Interaction (CLAPI $\left.{ }^{31}\right)$, élaboré par le groupe ICOR, regroupe 45 heures de parole dans des contextes variés comme les interactions professionnelles, institutionnelles, commerciales, didactiques ou médicales. Nous avons relevé 65 occurrences de notre forme. 
Concernant l'écrit, nous avons effectué des requêtes sur la partie écrite du Cerf. Nous avons collecté un corpus de presse écrite d'environ 130 occurrences sur 500000 mots ; les journaux consultés sont : Le Monde, le Monde diplomatique, l'Humanité, Le Nouvel Observateur et le journal satirique Charlie Hebdo. Enfin, nous avons consulté une autre tranche de 500000 mots issus de corpus divers, tels que des contes, des chansons ou encore des extraits de sketches humoristiques, dans laquelle nous observons une centaine d'occurrences.

\section{NOTES}

1. Voir Debaisieux (2006) pour «bien que, parce que et puisque» et Benzitoun (2006) pour " quand".

2. Voir annexe pour le détail des bases de données interrogées.

3. Comme l'indique Hava Bat Zeev : «La CS 'alors que' s'est développée à partir de l'adverbe temporel 'alors'. Le stade suivant dans l'évolution est l'apparition de la CS 'alors que'vers le treizième siècle comme synonyme de 'lorsque' ; (...) Toutefois, c'est la valeur adversative de cette CS qui se répand à partir de cette période. Le français contemporain fait usage de 'alors que' dans le sens temporel, adversatif et causal. » (Bat-Zeev Shyldkrot, 1987 :48).

Quand, alors que et tandis que, ont suivi le même parcours dans l'étoffement de leurs effets de sens.

4. Trésor de la Langue Française Informatisé, disponible sur : http://atilf.atilf.fr/tlf.htm

5. Il cite: «Je l'ai vue deux ou trois fois alors que j'étais à Toulouse » (Rob. Méthod, cité par Grevisse, 2008 : 1482).

6. M. Grevisse ne fait pas de distinction entre « alors que » et « tandis que » (2008: 1482).

7. Cf. Groupe Lambda (1975), avec des exemples comme : «tu es malade parce que tu as trop mangé » (259) (lien de dépendance syntaxique) vs. « il est malade parce qu'il a de la fièvre » (deux actes d'énonciation où la seconde construction verbale est une justification de la précédente) (263).

8. La syntaxe externe correspond à la relation établie sur la base de critères formels entre la « alors que construction » et le contexte dans lequel elle apparaît.

9. Ce point intéressant sera l'objet d'une étude plus vaste car l'emploi de cette forme semble très sensible aux genres des textes.

10. A l'intérieur de la rection, on distingue d'une part, les « éléments de valence » c'est-àdire les dépendants "qui servent à caractériser le sens et la construction minimale du verbe » (Blanche-Benveniste et al., 1990: 45) et d'autre part, les éléments de simple rection, que nous nommons « ajouts ».

11. Initialement présentée par C. Blanche-Benveniste et al. , dans l'ouvrage « Pronom et syntaxe » (1984).

12. Par « proformes », il est entendu des formes comme : ainsi, quelque part, comment... "A ce moment-là » et " quand » sont proportionnels à une "alors que construction » temporelle.

13. Toutefois, ce test n'a de fiabilité que s'il s'applique à des ajouts ; on ne peut reprendre par « et cela » des éléments de valence : ${ }^{*}$ il ressemble et cela à son père. 
14. Debaisieux (2006: 122) évoque les mêmes blocages avec le «bien que » régi : *c'est bien qu'elle soit confirmée par le séjour en Amérique hein dont on a déjà parlé qu'elle est insuffisante. Cela nous semble être également le cas pour la forme tandis que:? C'est tandis que j'étais à Toulouse que je l'ai vue deux ou trois fois.

15. «La non congruence est un type de modalité appréciative qui met obligatoirement en jeu deux entités et établit une relation d'anormalité entre elles. » (Guimier, $2000: 84$ ).

16. Les exemples précédés de * sont clairement jugés agrammaticaux, par contre pour le? précédant l'exemple, nous doutons de sa recevabilité car il ne se trouve pas de telles séquences dans les corpus.

17. La forme «mais » ne se pare pas du sémantisme argumentatif que parfois elle tire de la valeur propre de la phrase; nous ne sommes pas dans les situations que décrit Ducrot (1980: 16), lorsque « A mais $B$ » est interprétable comme : « A, tu as tendance à tirer de $A$ une certaine conclusion $r$; il ne le faut pas, car B, aussi vrai que A, suggère la conclusion non-r. ». Pour expliciter cela, on peut reprendre un exemple cité par Riegel (2008:619) : «Gavroche est petit, mais il est malin » où la première assertion oriente vers une certaine conclusion non formulée «il est vulnérable » mais où la seconde proposition introduit un argument plus fort.

18. Il s'agit d'un fonctionnement de type connecteur de discours entre deux énoncés qui est attesté pour d'autres conjonctions, comme puisque, mais qui s'oppose au fonctionnement comme élément périphérique antéposable à l'intérieur d'un même énoncé. (voir Debaisieux, 2006).

19. Dans ce paragraphe, il fait état de cette possibilité de changement modal pour la forme « alors que » et « tandis que ».

20. On pourrait aussi trouver ces mêmes effets avec « par contre » ou « en revanche ».

21. Nous faisons le même usage de cette notion que dans la rhétorique, à savoir deux constructions verbales qui développent les mêmes places syntaxiques.

22. Il utilise le terme de « cadratif » pour parler de ces pré-noyaux.

23. La syntaxe interne concerne la composition des unités en termes de catégories grammaticales à l'intérieur de la «alors que construction ».

24. Nous n'avons pas rencontré dans les corpus d'exemples avec «alors que ». Mais, «tandis que » a également la possibilité de réaliser ces phénomènes de projections.

25. Pour la différence entre relation « adversative » et « concessive » : « on voit maintenant se dessiner les contours de deux types de relations oppositives. La première est paradigmatique, symétrique et matérielle: nous l'appelons adversative. La deuxième est syntagmatique, asymétrique et logique : c'est la relation concessive. Un avantage évident de cette caractérisation est qu'elle prévoit l'existence de formes intermédiaires. » (Nolke, 1984 : 6)

26. En revanche, parce que n'est pas un bon équivalent : [21] * je comprends pas qu'on dise que Gutenberg a inventé l'imprimerie parce qu'il travaillait dans une imprimerie.

Cf. Groupe Lambda (1975) pour une distinction sémantico-pragmatique entre parce que et puisque.

27. Nous voyons en «dans ces conditions-là » une proforme en raison du caractère lexicalement très peu spécifié de cette tournure.

28. Dans des exemples du genre: «En Allemagne, ils ne mangent pas de lapin, alors qu'en Espagne, ils mangent du lapin» (exemple inventé), la négation n'a pas de portée englobante et s'arrête à la fin de la première construction verbale.

29. Dans cette structure, "la première partie comporte la formulation verbale, réalisée d'une façon qui crée une attente: un des éléments régis est réalisé sous une forme non lexicale, 
suspensive, qui laisse attendre une réalisation ultérieure sous forme de lexique; entre les deux, le verbe d'équivalence 'c'est'. » (Blanche-Benveniste et al., 1990 : 62).

30. Dans la négation polémique, « le locuteur s'oppose à une affirmation d'autrui, qu'il vise à réfuter. Il reprend dans son discours une affirmation qu'il attribue à autrui, que celui-ci l'ait formulée ou non, pour la nier. » (Riegel et al., $2008:$ 425).

31. Disponible sur : http://clapi.univ-lyon2.fr

\section{RÉSUMÉS}

Dans cet article, nous présentons les premiers résultats d'une analyse en cours qui porte sur la forme " alors que ». Dans les grammaires traditionnelles ou usuelles, son traitement en est réduit à sa plus simple expression : il ne se conçoit que sous le titre de «subordination ", lequel repose uniquement sur la présence d'une conjonction de subordination. Or, ce seul critère catégoriel s'avère insuffisant pour définir une telle relation syntaxique; la relation de dépendance entre deux constructions verbales doit être prouvée à partir d'une critériologie pertinente et non par la simple nature de l'élément introducteur. Nous proposons donc, grâce à des exemples attestés issus principalement de la langue parlée, de montrer les limites de la notion de « subordination » et de distinguer pour la forme "alors que" trois types grammaticaux remarquables par leur organisation syntaxique auxquels nous attribuerons des effets de sens précis.

This article presents the first results of an ongoing research devoted to the French conjunction "alors que". The traditional analysis usually conceives this grammatical form as a mere subordination marker. In our theoretical framework, the only presence of a "subordinating conjunction" does not prove in itself the existence of any genuine dependency relation in the utterance: we consider that syntactic dependency must necessarily correlate with several paradigmatic properties, such as the equivalence with a pronoun, the possibility to be cleft, and some other criteria. We will show that three main uses of "alors que" must be distinguished on a syntactic and semantic basis, and will illustrate our analysis with utterances mostly drawn from spoken corpora.

\section{INDEX}

Mots-clés : linguistique descriptive/de corpus, subordination/rection, approche pronominale, sémantique/pragmatique

Keywords : descriptive linguistics, subordination, pronominal approach, semantics / pragmatics

\section{AUTEUR}

\section{FANNY LAFONTAINE}

Aix-Marseille Université, LPL UMR 7309,

fanny.lafontaine@univ-amu.fr 